\title{
Adaptive Educational Games: Providing Non-invasive Personalised Learning Experiences
}

\author{
Neil Peirce, Owen Conlan, Vincent Wade \\ Trinity College, Dublin \\ \{peircen,Owen.Conlan,Vincent.Wade\}@cs.tcd.ie
}

\begin{abstract}
Educational games have the potential to provide intrinsically motivating learning experiences that immerse and engage the learner. However, the much heralded benefits of educational games seldom consider the one-size-fits-all approach to education they typically embody. The benefit provided by adaptive educational games is that of a motivating environment reinforced with a personalised learning experience. However, adapting a game to enhance its educational benefit endangers its intrinsic motivation and flow. This paper proposes a novel approach for non-invasively adapting a game to enable a personalised learning experience. This is achieved using an innovative, generic and reusable architecture, without mitigating the motivational features of gaming. An implementation of this approach in the form of the ALIGN (Adaptive Learning In Games through Noninvasion) system is detailed and the results of an authentic evaluation are discussed.
\end{abstract}

\section{Introduction}

Educational games can be seen as a progression in technology enhanced learning that provides direct support for a learner's motivation [1]. Although games can provide an intrinsically motivating experience, the complexities of educational game design is considerable [2]. With the full potential of educational games yet to be realized [3] one must consider the existing approaches to technology enhanced learning that have proven fruitful. For instance, adaptation has long proven beneficial in eLearning as is evident in Adaptive Hypermedia [4, 5]. Combining adaptation and educational games can uniquely present a personalised supportive motivational experience. In realising this motivation through appropriate challenge, curiosity, fantasy, and control [6] there remains great potential to address the under-motivated learner.

The continuing progression in educational gaming has seen a move away from the crude separation - or
Shavian reversals [7] - of gaming and learning scenarios present in first generation educational games towards a more integrated gaming and learning experience. (e.g. Darfur is Dying, Peacemaker, Brain Training). One notable characteristic of these contemporary games is the emphasis placed on maintaining an enjoyable gaming experience, which is often prioritised over the regularity and frequency of learning content. Whereas this may initially seem a misguided approach in consideration that a positive learning outcome is the ultimate goal, one must consider that the effect of doing the opposite, i.e. prioritising learning content over gaming, is considerably worse. In a scenario where the presentation of the learning content is prioritised over the gaming experience, the possibility of the gaming experience being negatively impacted increases significantly. Without an immersive gaming experience the benefits of using games as a motivational vehicle for learning becomes compromised. It has been identified by a number of authors [3,8] that an educational game must be a game first and an educational tool second. Without this prioritisation the potential benefits of gaming are mitigated.

Although a learning experience that is intrinsically motivating is advantageous, it is but one of many contributing factors that can lead to effective learning outcomes. The field of adaptive hypermedia in particular has long focused on another factor, the benefits provided by the adaptability and personalisation of the learning experience.

This paper addresses the problem of non-invasively supporting a learner within an adaptive educational game. Through an innovative approach to personalising learning challenge, and meta-cognitive support, it is shown how an immersive 3D adventure game can be personalised in a manner that is not invasive to the player's gaming experience. Explicitly, the non-invasive adaptations do not compromise the game narrative and character consistency; they are non-invasive to the gameplay experience. This approach promotes augmentation over intervention in 
adapting existing educational game content, whilst avoiding the complexity of adaptive narratives by noninvasively providing this augmentation. Additionally through focussing on adapting challenge the approach aims to further aid motivation, a significant factor in positive learning outcomes [9].

This paper details a reusable architecture that supports the approach. A real-world integration and authentic trial of the system with an educational game is evaluated with a discussion on the success of the approach and on future work.

This paper starts with an overview of the state of the art in adaptive educational games, detailing the current approaches and examining existing systems. The paper then details the design of the ALIGN system and the rationales behind our approach to providing educational adaptation. The third section examines the implementation of the ALIGN system and its initial evaluation using the ELEKTRA game [10]. The final section of this paper summarises the findings from the initial experimental evaluation and presents a short discussion on the success of the approach and future work.

\section{Adaptive Educational Games}

The challenge of integrating personalised learning experiences into educational games provides significant challenges to an area of research that is only now making progress away from earlier 'Shavian Reversals' [7]. In many instances little was done to blur the boundaries between gaming and learning, something which is considered a desirable feature [11]. Whilst research into the effective integration of gaming and learning is still ongoing $[8,12]$ the compulsion to provide a personalised educational experience is driven by established research in Intelligent Tutoring Systems (ITS) [13] and Adaptive Hypermedia [14]. The provision of personalisation is long established to be beneficial to learning outcomes and experience $[4,5]$. Whereas ITS and AH systems typically have complete control of the learning experience, adaptive educational games (AEGs) are faced with the additional hurdle of maintaining game narratives which potentially may be static and linear. As a result any adaptation performed in an AEG must be appropriately constrained.

The potential thus remains that through the personalisation of the learning experience within educational games, the intrinsic motivation provided by games can be complemented with a tailored learning experience.

Artificial Intelligence and adaptation within digital games is in itself an active area of research focussing on such areas as Dynamic Difficulty Adjustment, path finding, and NPC (Non Playing Character) behaviour $[3,15]$. Although the burgeoning games industry has spurred such AI in realising commercial applications, the usage of AI techniques within adaptive educational games remains limited. The games that have implemented such adaptations have had to address the same hurdle as faced when integrating education into games, that is, how to integrate education within a game without destroying the gaming experience.

Such games as the DARPA funded Tactical Language \& Cultural Training System (TLCTS) [16] have shown that effective learning outcomes can be achieved through the use of adaptive educational games [16]. The realisation of these outcomes however required considerable development costs and the use of sophisticated natural language processing and multimodel adaptation. Such monetary and technical requirements are contributory factors to the scarcity of such games. One attempt to address the associated development cost can be found in the <e-adventure> framework [17] that provides a simplified method of developing adaptive educational games that can also be integrated into existing Learning Management Systems (LMS). Although the <e-adventure> framework simplifies the creation of adaptive educational games, the output game genre is limited to 2D point and click, a limiting feature in consideration of the aptness of particular game genres for particular learning content as is highlighted by Prensky [8].

One of the key motivational factors found within games is a strong storyline [18], a tool which is often utilised within educational games. Whereas integrating educational content within a motivating narrative is a challenging task, the further complexity added when considering adaptive educational content is one of the considerable challenges facing adaptive educational games. Despite the challenges of developing adaptive narratives, such games as Façade [19] have shown how a compelling game narrative can provide an immersive experience. Whilst Façade was not promoted explicitly as an educational game there exist educational examples such as Virtual Team Collaborator (VTC) [20] that adopt a similar approach to adaptive narratives. Although successful in their technical approach, both Façade and VTC exemplify the considerable complexity in implementing adaptive narratives that require the authoring of multitudes of differing narrative strands.

Despite the commonality of narratives in educational games there exist other methods of creating a motivating gaming experience. The Prime Club/Climb game [21] for instance utilises an adaptive pedagogical agent to provide hints, guidance and motivational support in place of a storyline. Through a system of real-time assessment the Prime Club game 
ensures that the adaptation provided through the pedagogical agent is always appropriate and is tailored to providing a motivating learning experience.

Whereas the instances of adaptive educational games continue to increase they are still few in comparison to the growing number of non-adaptive educational games. In consideration of this, one approach is to extend existing educational games with adaptive educational content. Such an approach was taken with the Ecotoons 2 [22] game that extended the existing Ecotoons game. Approaches such as this have shown promising results in the feasibility of using adaptation as an enhancing feature to educational games.

While games such as Façade and TLCTS manage to integrate adaptation into the game in a seamless manner, the approaches taken are not without their drawbacks. In both cases the complexity of the implementation was considerable. Not withstanding the benefits of adaptive narratives, an approach to augment existing educational games without adapting narratives is feasible and can present reduced complexity. Further to this a common feature of the existing adaptive educational games is the tight integration of the educational adaptation with the game. As a consequence the educational adaptation becomes tightly bound to a specific game hindering the potential reuse.

\section{Design \& Architecture}

The conceptual separation between gaming and educational adaptation underpins the ALIGN system. Although game logic and adaptation logic can be disparate in nature, overlaps remain in such areas as the preservation of a flow experience [23]. The constituents of a flow experience can be found in both gaming and educational adaptation. In particular the balancing of challenge and skills is a feature important to both games [18] and learning [24].

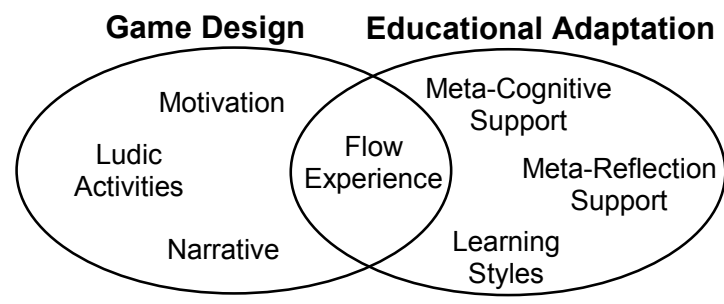

Figure 1 - Separation of concerns

Despite this overlap the educational adaptation remains largely agnostic to the underlying game. It is feasible to consider that common axes of adaptation such as motivational support, meta-cognitive feedback, and meta-reflective feedback can all be achieved irrespective of the underlying game. In consideration of this there exists the possibility that adaptation authored at a level abstracted from a particular game could be reused within multiple games.

In order to effectively use abstracted adaptation it is necessary to map between game specificities and the abstracted logic. Within the ALIGN system this is achieved firstly through a process of inference that translates game specificities to abstract educational concepts, secondly through a process of realisation that translates abstract adaptations to game world modifications. A typical example of inference would be the mapping of a player's failure during a game task to the decrease in the skill related to the task [25]. The process of realisation in this case would translate the abstract desire to aid the player's skill acquisition into a game world NPC offering verbal guidance.

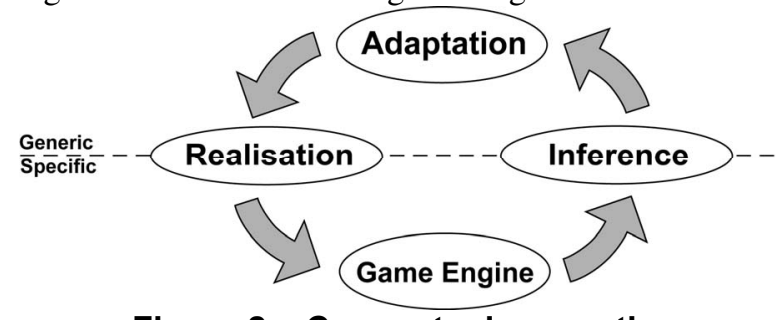

Figure 2 - Conceptual separation

The separation of the adaptation and the game features is at the core of the ALIGN system. This approach allows the adaptation and the game logic to operate independently and also to be authored independently. This feature aims to reduce the cost of implementing adaptation through maximising the reuse of domain expert authored adaptation.

Each adaptation is represented as an identifier and a text string describing the envisaged educational outcome. Each one of the adaptations is referred to as an Adaptive Element (AE) and is further annotated with meta-data describing the game scenarios where it can be appropriately used. An example of AE would consist of a description such as 'motivating hint for skill $\mathrm{X}$ ' and would be realised as an encouraging verbal hint being given by an NPC relating to skill X.

This approach allows the educational adaptation to be unconcerned with how an $\mathrm{AE}$ is realised and further decouples the ALIGN system from a specific game, thus allowing for greater independent authoring of each.

Whereas rules can be used to select desirable game adaptations, the range of possible AEs must first be constrained. It is this constraint that ensures only appropriate changes are made within the game. The constraint of the Adaptive Elements is conceived as a two stage process based on the game feasibility and on 
the game appropriateness. The game feasibility constraints eliminate all AEs that are not available due to the current game state and context. For example if an NPC was not present then many AEs would not be possible. The appropriateness constraint is the crucial component of the non-invasive architecture as it allows for the AEs to be constrained based on factors such as desirable NPC behaviour and game narrative consistency. Typical consistency constraints ensure that NPCs do not repeat or contradict themselves in the dialogs they have with the player. Such repetitive or contradictory actions are identified by maintaining a history of all adaptations used.

The advantage this approach provides is that the adaptation logic is considerably simplified. As the AEs are already constrained by feasibility and appropriateness, the adaptation logic can solely focus on selecting desirable AEs.

Interface Between the Game and the Adaptation. To achieve non-invasive educational adaptation the game need only satisfy two requirements. Firstly the game must expose all game evidence pertinent to each adaptation offered by the game, such as task success/failure or character movement. Secondly the continuously propagating through the four processes. In order to achieve effective non-invasive adaptation the intervention constraint process relies heavily on the game and adaptation context that has been accumulated. Figure 3 illustrates the components of the architecture and their interactions.

The four conceptual processes labelled one to four are detailed below. Details of the key components involved in these processes are also given.

1. Inference of raw game evidence into facts suitable for educational adaptation.

2. Accumulation of inferred evidence, game state, and previous adaptation to develop a temporally holistic view of a learner's game experience.

3. Refinement of candidate adaptations based on game context, and previous adaptations to ensure the use of non-invasive adaptations.

4. Selection of adaptation based on abstracted pedagogical rules and appropriately refined candidate adaptive elements.

In order to achieve non-invasive adaptation the architecture relies on the inputs of both game designers and learning domain experts. The division of responsibility starts with the game designer having sole responsibility for the 'Game Constraints', 'Consistency

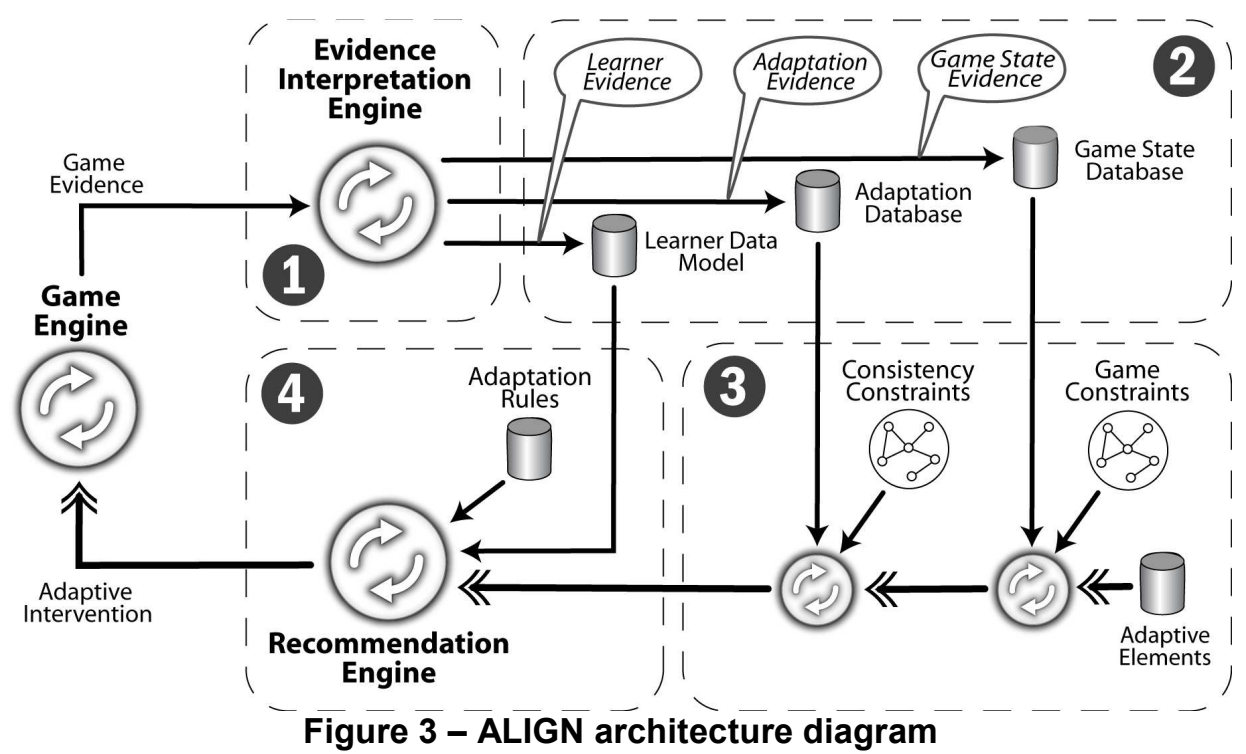

game must share a set of Adaptive Elements with the system and the game must be receptive to activating an $\mathrm{AE}$ when requested.

Architecture. The ALIGN system architecture is divided into four conceptual processes; inference, context accumulation, intervention constraint, and adaptation realisation. The entire ALIGN system operates in a continuous and cyclical manner with data
Constraints' components. The learning domain experts having sole responsibility for the 'Adaptation Rules'. The 'Evidence Interpretation Engine' and 'Adaptive Elements' requires contributions from both parties.

The following sections detail the functionality provided by each of the architectural components. Importantly each of the components can be modified independently without necessarily requiring modification to other connected components. This 
feature is of particular benefit where new adaptive elements can be authored and automatically used by the adaptation rules.

Evidence Interpretation Engine. This component uses a rule base and other probabilistic methods to translate game specific events to facts that can be used for educational adaptation. Methods such as Knowledge Space Theory (KST) (see first experiment) can be used to infer current learner skills. The collaboration of domain experts and game designers is necessary to effectively translate the significance of game specific data.

Game Constraints. Through the use of rules governing which Adaptive Elements are available in different scenarios, this component ensures that only Adaptive Elements that are possible in the current game context are available for selection.

Consistency Constraints. The importance of this component is in its ability to prevent the selection of Adaptive Elements that would be invasive to the player's game experience. The rules governing this component are strongly influenced by the game designer to ensure narrative consistency and the appropriate behaviour of NPCs.

Adaptive Elements. In order for game specific adaptations to be made a record of all of the game's Adaptive Elements is used. Each element is annotated with meta-data describing the game scenarios in which it can be used, and the abstract outcome that is envisaged through using the adaptive element.

Adaptation Rules. This component performs the adaptation based on a set of rules that examine the learner model and determine the desired adaptation outcome. A suitable adaptive element (if available) is then chosen from the already constrained adaptive elements. The Adaptive Elements are selected based on the outcome they are annotated with.

Technical Implementation. The ALIGN system is implemented in Java 1.5 and utilises the JBoss Drools rule engine at its core as an efficient method of executing declarative rules. All communication between game engines and the ALIGN system is currently achieved using TCP/IP sockets. All inferred evidence and Adaptive Elements within the system are inserted into a Drools working memory where they are manipulated by the Adaptation rules, Consistency Constraint rules, and Game Constraint rules. The ordering of rule execution is governed by rule flows, agenda groups, and rule prioritisation. The Learner Data Model, Adaptation Database, and Game State Database are implemented as sets of facts within the working memory. The facts are ordered chronologically allowing them to be accessed as needed for AE constraint and selection. The Adaptive
Element descriptions are authored as an XML document which is parsed by the ALIGN system to create facts representing the AEs. These facts are then inserted into the working memory.

\section{The ELEKTRA game}

The initial experiment using the ALIGN system sought to provide the educational adaptation for the technical demonstrator component of a European Commission funded FP6 project called ELEKTRA [10]. The aim of the ELEKTRA project was to revolutionise technology-enhanced learning through merging expertise in cognitive science, pedagogical theory, computer science and neuroscience with the innovations of computer gaming, design and development.

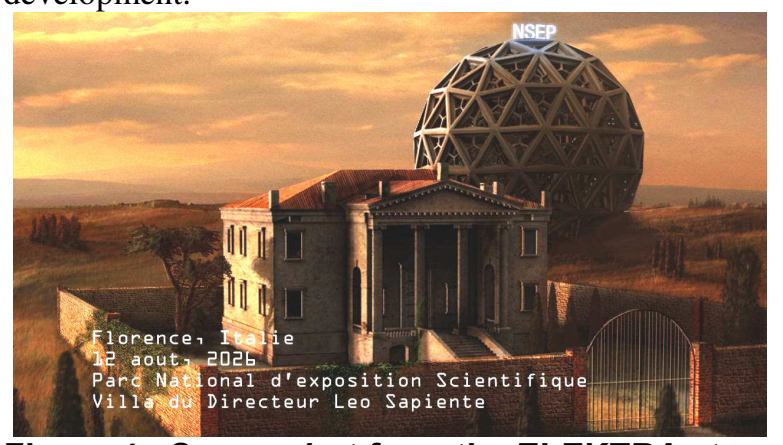

Figure 4 - Screenshot from the ELEKTRA story

The demonstrator component of ELEKTRA is a first-person 3D role-playing adventure game. It features a motivating storyline, interactive non-player characters, educational adaptation, and a rich visual environment. The educational content of the game focuses on the physics of optics as studied by 13-15 year old students under the French state curriculum. The ELEKTRA game tightly integrates gaming, learning, and storyline to create an immersive experience whereby the boundaries between gaming and learning are blurred.

The ELEKTRA game places the player in the role of George the nephew of a kidnapped scientist. In order to rescue his uncle George must overcome challenges and prove his abilities to an interactive NPC represented by the ghost of Galileo Galilei. The challenges involved are all pertinent to the game's storyline as well as encouraging learning through experimentation.

Due to constraints within the project the number of learning tasks developed was restricted. For this reason the possibilities for Macroadaptivity, the adaptation of challenge ordering and selection was not practical. As a consequence of this all adaptation provided is Microadaptive, and achieved entirely within learning 
tasks. The adaptation within ELEKTRA focuses on two areas (1) motivational and hinting support, and (2) meta-cognitive feedback. In order to provide motivational and hinting support an additional system component, the Skill Assessment Engine (SAE), was added to the inference process to calculate probabilistic values for the learner's skills based on their actions. This system utilises a real-time implementation of Knowledge Space Theory (KST) and is further detailed in [25].

The following example illustrates the typical process involved in delivering non-invasive affective/motivational feedback within the ELEKTRA game. In this example the player has been experimenting with an apparatus known as the slope device that is designed to show the effects that magnetism, gravity, and wind can have on materials of varying composition and density. The player must manipulate a magnet and fan to get a marble (wooden, iron or hollow plastic) into a target area under the supervision of an NPC named Galileo Galilei. The stages in the adaptation process are given below.

1. Message received from the game engine indicating a hollow plastic marble has missed the target.

2. The skills associated with this action are inferred and the skill probabilities are updated by the SAE accordingly.

3. The changes in skill probabilities are added as facts to the learner model.

4. The appropriate adaptive elements to be used are constrained in consideration of the presence of Galileo and the previous adaptations given through the Galileo NPC.

5. Using the adaptation rules it is identified that there have been three successive decreases in the probability for the skill "Knowledge of what a hollow object is and that it is less heavy than a solid object". Based on this, the desirable outcome of encouraging this skill is selected.

6. A suitable adaptive element is chosen from the constrained adaptive elements and is sent to the game engine.

7. The game engine executes the adaptation; in this case the adaptation consists of the Galileo NPC saying "Yes. It isn't easy, and I'm not sure that I would do any better in your position, but you must persevere."

8. Feedback that the adaptation was successfully used is sent to the ALIGN system.

Due in part to the flexibility of the ALIGN system it became possible to integrate seven different types of adaptation into the ELEKTRA game. By freeing the domain experts from concerns regarding the invasiveness of adaptations, more effort could be focused on the variety and complexity of the adaptation provided. This resulted in the following diverse range of microadaptive interventions being provided to the ELEKTRA game -

- Cognitive feedback

- Instant meta-cognitive feedback

- Tendency meta-cognitive feedback

- NPC Confidence/Prudence feedback

- Affective/motivational feedback

- Knowledge based hinting

- Progression hinting

Given space restrictions it is not feasible to elaborate scenarios for each adaptation type, however further examples of NPC hints include; "You're successful in finding the solution. But you are not showing me that I can trust your judgment" (Tendency meta-cognitive), "Wood is a medium-weight and nonmagnetic material. It is not influenced by the magnet, but can be influenced by the fan" (Knowledge based hinting).

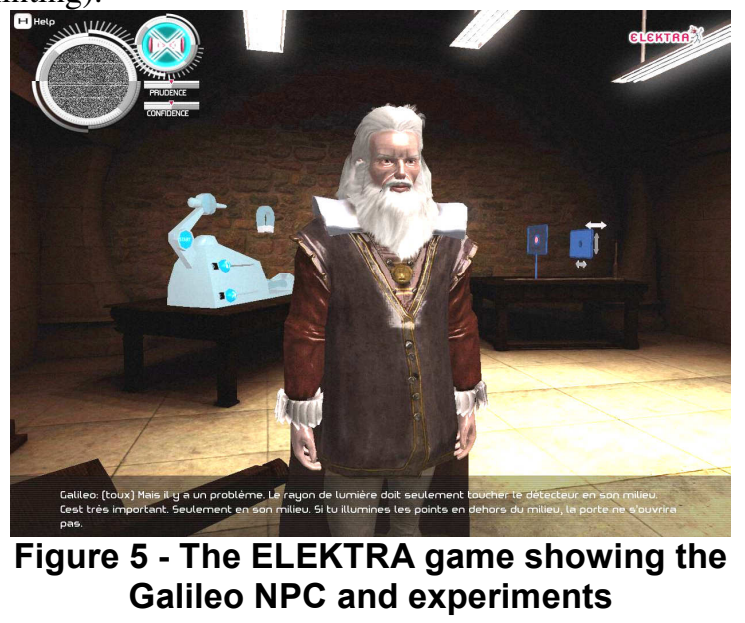

Adaptation Setup. The adaptation setup for the ELEKTRA game required a real-time implementation of KST as part of the SAE [25]. This component calculated the skill probabilities of 25 skills from 12,414 skill states. The setup additionally contained 65 adaptive rules, an 8 stage rule flow, and 197 Adaptive Elements spread over 3 learning scenarios. Through a high performance Java implementation typical response times were less than $50 \mathrm{~ms}$ providing a responsive gaming experience.

Experimental Setup. The experiment using the ELEKTRA game had the objectives to determine (1) the perceived invasiveness of the adaptation, (2) the effectiveness of challenge personalisation, and (3) the motivational impact of the adaptations provided. Due to the subjective nature of the objectives being 
evaluated, a largely qualitative evaluation was appropriate. The one exception to this was for the second objective where a combination of qualitative and quantitative data was used.

In order to achieve the above objectives, the ELEKTRA game was evaluated with 49 students where the learning content formed part of their assessable curriculum. On average the students took 30 minutes to complete the tasks. The experiments took place in late January 2008 at a number of schools in and around Paris under the supervision of the ELEKTRA partner ORT France ${ }^{1}$. Each experiment was preceded by a questionnaire designed to gauge the student's competency in the learning topics covered. A post questionnaire was also used to assess learning impact, and to assess qualitative player experience in terms of game difficulty, flow experience, and the perceived invasiveness of adaptations. Comprehensive logs were also automatically recorded by the ALIGN system that detailed every action taken by the user within the game and every adaptation performed.

\section{Experimental Results}

In terms of the perceived invasiveness of the adaptation provided the success of the approach was assessed through direct questions relating to the aptness of the adaptations provided and indirectly through the overall flow experience of the learners.

With the majority of the noticeable adaptations being delivered through the Galileo NPC, the perceived appropriateness remained consistent between adaptive hints and static hints. Similarly the flow experience was not adversely affected, indicating that in the least the ALIGN approach maintains the existing flow experience.

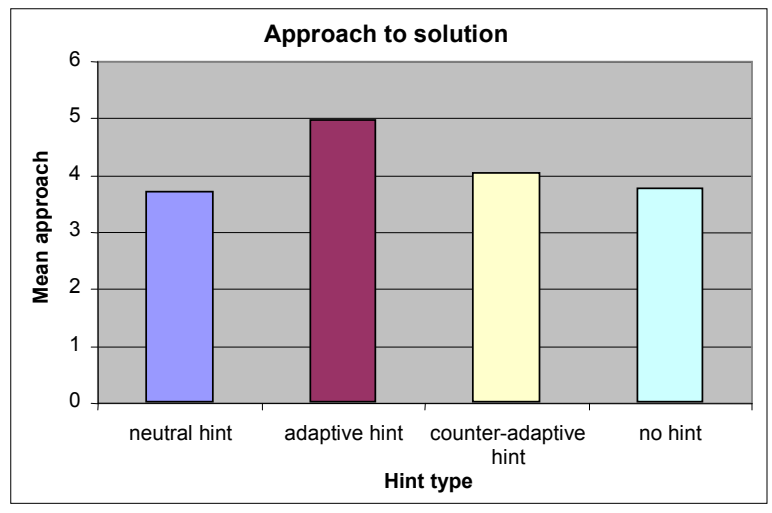

Figure 6 - Mean Approach to Correct Result Following Hint Type

\footnotetext{
${ }^{1}$ ORT France is a French non-governmental education and training organisation, http://www.ort.asso.fr/
}

To assess challenge personalisation and consequently motivation, the impact of adaptive hints were assessed. In particular, it was found that following an experiment failure by a player, a subsequent adaptive hint improved the player's subsequent approach to the correct solution, see Figure 6 . The metric of approach is the difference in absolute distance to the correct solution based on two subsequent attempts. The attempts were made using the experiments configurable scale of 1-100. For this experiment three groups of students were used, two of which were control groups which received no hints or pre-scripted hints, respectively. The third group received the adaptive hints.

A second aspect of the evaluation used a combined median-split analysis to compare the 'low adaptivity' and 'high adaptivity' extreme groups. The results of this analysis showed the "High Adaptivity" group reported:

- Higher amount of invested effort and a higher degree of absorbedness

- Higher relatedness with the Galileo NPC

- Higher usefulness of the slope device

- Higher confidence in their own learning achievement

- Easier handling (lower Extraneous Cognitive Load) of the slope device

For this analysis the 'Low Adaptivity' group was defined as having $54 \%$ or less adaptive interventions and more than $27 \%$ counter-adaptive interventions. The 'High Adaptivity' group was defined as having more than $54 \%$ adaptive interventions and only $27 \%$ or less counter-adaptive interventions.

Due to human error in recording dialogues, defining adaptive elements, and inaccurate consistency constraint rules; counter-adaptive hints were given to learners. These hints typically appeared to be somewhat out of context. A typical example would be a hint relating to the density of an iron marble when dealing with a plastic marble. Additionally neutral hints were identified as being hints that did not make reference to the current context but were considered correct.

Despite the evaluation limitations imposed, significant results were obtained for the evaluation of the ELEKTRA game. Whilst not statistically significant the learning outcome and flow experience for the ELEKTRA game with adaptive hints was positive and encouraging with the significance of the results mitigated due to the small sample size. 


\section{Conclusions and Future Work}

The realisation of adaptive educational games presents financial, integration, and technical challenges that have to date hindered the progress of this field of technology enhanced learning. Through the ALIGN system we have demonstrated a novel approach to adaptive educational games that aims to minimize the gaming impact of the adaptation whilst enabling reuse of the adaptation logic. Consequently where reuse can be maximised the development costs of adaptive educational games can be reduced.

The authentic evaluation of the ALIGN system has shown positive results in our initial experimentation and represents a step forward in adaptive educational games. However, the need to examine the generality of the approach in consideration of differing game genres, subject areas, and the feasibility adaptation reuse is required. Due to technical and language constraints the ELEKTRA game presented evaluation difficulties that limited the sample set. Additional experiments using the ALIGN system are currently being developed that will utilise a more portable game platform, in this case Adobe Flash, in order to facilitate the evaluation of the system with a larger sample set. The provision of authoring tools to for the ALIGN system is also a consideration for our future work.

This research was initially funded by the ELEKTRA project as part of the European Commission's FP6 IST programme, Contract No. 027986 [10]. This research is part funded by Science Foundation Ireland through the CNGL project, www.cngl.ie

\section{References}

[1] L. P. Rieber, "Seriously considering play: Designing interactive learning environments based on the blending of microworlds, simulations, and games," Etr\&D-Educational Technology Research and Development, vol. 44, pp. 43-58, 1996.

[2] G. K. Akilli, "Games and Simulations: A New Approach in Education?," in Games and Simulations in Online Learning: Research and Development Frameworks, D. Gibson, C. Aldrich, and M. Prensky, Eds.: Information Science Pub., 2007, pp. 1-20.

[3] R. Van Eck, "Building Artificially Intelligent Learning Games," in Games and Simulations in Online Learning: Research and Development Frameworks, D. Gibson, C. Aldrich, and M. Prensky, Eds.: Information Science Pub., 2007, pp. 271-307.

[4] P. Brusilovsky, "Methods and techniques of adaptive hypermedia," User Modeling and User-Adapted Interaction, vol. 6, pp. 87-129, Jul 1996

[5] O. Conlan and V. P. Wade, "Evaluation of APeLS - An adaptive eLearning service based on the multi-model, metadata-driven approach," Adaptive Hypermedia and Adaptive Web-Based Systems, Proceedings, vol. 3137, pp. 291295, 2004.
[6] T. W. Malone and M. R. Lepper, "Making learning fun: A taxonomy of intrinsic motivations for learning," Aptitude, learning, and instruction, vol. 3, pp. 223-253, 1987.

[7] S. Papert, "Does Easy Do It? Children, Games, and Learning," Game Developer, vol. 5, p. 88, 1998.

[8] M. Prensky, Digital Game-Based Learning: Paragon House, St. Paul, Minnesota, USA, 2001.

[9] M. R. Lepper, "Motivational Considerations in the Study of Instruction," Cognition and Instruction, vol. 5, pp. 289-309, 1988.

[10] "ELEKTRA - Enhanced Learning Experience and Knowledge TRAnsfer", Retrieved 25th June 2008 from http://www.elektra-project.org

[11] M. Pivec, "Editorial: Play and learn: potentials of game-based learning," British Journal of Educational Technology, vol. 38, pp. 387-393, 2007.

[12] R. Van Eck, "Digital Game-Based Learning: It's Not Just the Digital Natives Who Are Restless," EDUCAUSE Review, vol. 41, pp. 16-30, 2006

[13] E. Wenger, Artificial intelligence and tutoring systems: computational and cognitive approaches to the communication of knowledge: Morgan Kaufmann Publishers Inc. San Francisco, CA, USA, 1987.

[14] P. Brusilovsky, "Adaptive Hypermedia," User Modeling and User-Adapted Interaction, vol. 11, pp. 87-110, 2001.

[15] D. Charles, "Enhancing Gameplay: Challenges for Artificial Intelligence in Digital Games," in 1st World Conference on Digital Games, University of Utrecht, The Netherlands, 2003.

[16] W. L. Johnson, N. Wang, and S. Wu, "Experience with serious games for learning foreign languages and cultures," in SimTecT Conference., Australia, 2007.

[17] P. Moreno-Ger, D. Burgos, J. L. Sierra, and B. FernándezManjón, "A Game-Based Adaptive Unit of Learning with IMS Learning Design and <e-Adventure>. ," in Second European Conference on Technology Enhanced Learning (EC-TEL 2007), Crete, Greece., 2007.

[18] K. Salen and E. Zimmerman, Rules of play: game design fundamentals: MIT Press, 2003.

[19] A. Stern and M. Mateas, "Build It to Understand It: Ludology Meets Narratology in Game Design Space," in International DiGRA Conference Vancouver, British Columbia, Canada 2005.

[20] S. Franziska and I. Tsuyoshi, "Combining personalisation and adaptation in game-based learning systems," in Proceedings of the sixth conference on IASTED International Conference Web-Based Education - Volume 2 Chamonix, France: ACTA Press, 2007.

[21] C. Conati, "Probabilistic Assessment of User's Emotions in Educational Games," Applied Artificial Intelligence, vol. 16, pp. 555-575, 2002.

[22] R. M. Carro, A. M. Breda, G. Castillo, and A. L. Bajuelos, "A Methodology for Developing Adaptive Educational-Game Environments," in AH 2002:Adaptive Hypermedia and Adaptive Web-Based Systems Malaga, Spain: Springer, 2002.

[23] M. Csikszentmihalyi, Flow: The psychology of optimal experience. NY: New York: Harper and Row., 1990.

[24] D. J. Shernoff, M. Csikszentmihalyi, B. Schneider, and E. S. Shernoff, "Student engagement in high school classrooms from the perspective of flow theory," School Psychology Quarterly, vol. 18, pp. 158-176, Sum 2003.

[25] D. Albert, C. Hockemeyer, M. D. Kickmeier-Rust, N. Peirce, and O. Conlan, "Microadaptivity within Complex Learning Situations - a Personalized Approach based on Competence Structures and Problem Spaces," in 15th International Conference on Computers in Education, ICCE 2007 Hiroshima, Japan, 2007. 